\title{
Spin-sensitive interference due to Majorana state on interface between normal and superconducting leads
}

\author{
J. Barański, ${ }^{1}$ A. Kobiałka, ${ }^{2}$ and T. Domański ${ }^{2, \text { * }}$ \\ ${ }^{1}$ Institute of Physics, Polish Academy of Sciences, 02-668 Warsaw, Poland \\ ${ }^{2}$ Institute of Physics, M. Curie Sktodowska University, 20-031 Lublin, Poland
}

(Dated: October 5, 2018)

\begin{abstract}
We investigate the subgap spectrum and transport properties of the quantum dot on interface between the metallic and superconducting leads and additionally side-coupled to the edge of topological superconducting (TS) chain, hosting the Majorana quasiparticle. Due to chiral nature of the Majorana states only one spin component of the quantum dot electrons (say $\uparrow$ ) is directly affected, however the proximity induced on-dot pairing transmits its influence on the opposite spin as well. We investigate the unique interferometric patterns driven by the Majorana quasiparticle that are different for each spin component. We also address the spin-sensitive interplay with the Kondo effect manifested at the same zero-energy and we come to conclusion that quantum interferometry can unambiguously identify the Majorana quasiparticle.
\end{abstract}

PACS numbers: 74.45.+c,73.23.-b,73.22.-f,73.21.La

\section{INTRODUCTION}

Many-body effects can generate in condensed matter systems a plethora of either bosonic (like phonons, magnons) or fermionic quasiparticles (e.g. polarons). Recently enormous activity has been devoted to very exotic type of quasiparticles, resembling the Majorana fermions ${ }^{4-8}$ that are identical with their own antiparticles. Such emergent quasiparticles appear under specific conditions in the symmetry broken states $\frac{1-3}{-3}$ and their non-Abelian character makes them of interest for quantum computing and/or brand new spintronic devices 9 .

Realization of the Majorana quasiparticles has been predicted in various systems, for example in: vortices of superfluids 10 , three-dimensional11 ${ }^{11}$ or twodimensiona112 topological insulators coupled to superconductors, noncentrosymmetric superconductors 13 , electrostatic defects in topological superconductors ${ }^{14}$, $p$-wave superconductors 15 , the semiconducting 16,17 or ferromagnetic ${ }^{18}$ nanowires with the strong spin-orbit interaction coupled to $s$-wave superconductors, Josephson junctions ${ }^{19}$, ultracold atom systems ${ }^{20}$, and other. Experimental evidence for the Majorana quasiparticles has been already reported by the tunneling spectroscopy using the Rashba nanowires coupled to the bulk $s$-wave superconductors ${ }^{21-24}$. Zero-bias enhancement of the differential conductance observed at the edges of such wires $^{21-23}$ has been interpreted as signature of the Majorana mode, but similar feature can be eventually assigned to disorder ${ }^{25}$, Kondo effect in a crossover from the doublet to singlet configurations ${ }^{26,27}$ or other effects 28 .

For unambiguous identification of the Majorana quasiparticles there have been proposed several alternative methods, relying e.g. on optomechanical detection ${ }^{29}$, shot noise measurements ${ }^{30}$, Josephson spectroscopy ${ }^{31}$ using heterostructures comprising the quantum dot (QD) side-attached to the nanowire (see Fig. 11). In the case when both external leads are metallic it has

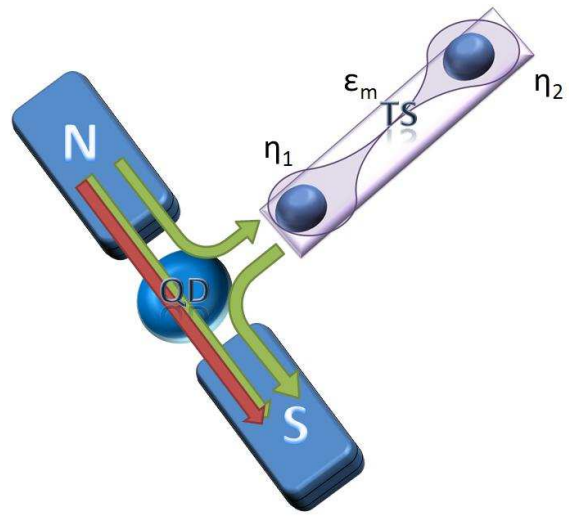

FIG. 1: Schematic illustration of the quantum dot (QD) laterally coupled to the metallic $(\mathrm{N})$ and superconducting $(\mathrm{S})$ electrodes and additionally hybridized with the Rashba nanowire, hosting the Majorana quasiparticles $\eta_{1}$ and $\eta_{2}$ at its edges. Green arrows indicate possible tunneling routes of $\uparrow$ electrons and the red arrow corresponds to $\downarrow$ electrons.

been predicted reduction (by half) of the quantum dot conductance $^{32}$, suppression of the Seebeck coefficient (due to perfect particle-hole symmetry at the Fermi level) ${ }^{33}$ and unique interferometric lineshapes ${ }^{34}-39$.

T-shape setup, where QD is laterally coupled between the superconducting and metallic leads (Fig. 1) can reveal the fingerprints of Majorana fermions in the subgap spectrum 40 . This configuration was already addressed in the literature ${ }^{40-42}$ but the spin-sensitive transport properties have not been analyzed in detail. Charge transport can occur at low voltage via the Andreev scattering, when electrons from the normal lead are converted into the Cooper pairs of superconductor reflecting the holes back to the same metallic electrode. Such spinselective Andreev spectroscopy has been suggested for probing the vortices in topological superconductors 43,44 and it has recently provided evidence for the Majorana modes in $\mathrm{Bi}_{2} \mathrm{Te}_{3} / \mathrm{NbSe}_{2}$. 
Two normal quantum dots arranged in the same Tshape configuration (as shown in Fig. 1) have been earlier studied by us $\frac{47}{4}$ and other groups $\frac{48-51}{-5}$. These studies indicated that quantum interference effects are capable to probe an interplay between the electron paring (manifested by Andreev/Shiba states) and the strong correlations. Here we extend the previous analysis 47 , by considering the influence of side-attached Majorana quasiparticle on the spin-resolved subgap spectrum of central QD and the transport properties. We show that Andreev transport would reveal interferometric lineshapes driven by the Majorana quasiparticles. Furthermore, we discuss how such interferometric features combine with the Kondo effect that is manifested at the same zero-energy.

The paper is organized as follows. In Sec. II we formulate the microscopic model and study interferometric effects appearing in a subgap spectrum of the uncorrelated QD. Next, in Sec. III, we analyze the correlation effect for the Kondo regime. Summary and conclusions are listed in Sec. IV. Some helpful technical details are presented in the Appendices.

\section{FORMULATION OF THE PROBLEM}

Due to chiral properties the ends of topological superconducting wire, that host a pair of Majorana fermions, are spin polarized ${ }^{52-54}$. For this reason we assume that only one spin the central quantum dot (QD) in the Tshape configuration (Fig. 1) is directly coupled to the Majorana quasiparticle 55 . When both electrodes are conducting the spin $\uparrow$ and $\downarrow$ transport channels would be independent, at least in absence of the correlations 56 . This is however no longer true, if one (or both) lead(s) is (are) superconducting, because of the proximity effect which mixes the particle with hole degrees of freedom ${ }^{57,58}$. In consequence, any physical process that engages electrons of a given spin simultaneously affects its opposite spin partner ${ }^{59}$. Such mechanism will prove to be important when considering the quantum interference driven by the side-coupled Majorana quasiparticle.

The previous study $\frac{47}{4}$ indicated that electron pairing induced in the normal double quantum dot (DQD) on interface between the metallic and superconducting electrodes is characterized by two lineshapes: Fano-type resonance formed near the energy $\epsilon_{2}$ of the side-coupled quantum $\operatorname{dot} 60$ and anti-Fano structure appearing at $-\epsilon_{2}{ }^{47}$. These features are detectable in the subgap Andreev conductance. In the present study we check whether similar effects appear when the central quantum dot is coupled to the Majorana quasiparticle, whose generic nature is related to only one spin (say $\uparrow$ ). For clarifying the interferometric lineshapes appearing in the spectrum of QD and the Andreev transport we briefly revisit the usual N-DQD-S heterostructure, imposing the spin polarized inter-dot hopping (Appendix B). Such consideration provides useful interpretation of the spindependent Fano and anti-Fano resonances.

\section{A. Microscopic model}

Tunneling structure, comprising the central QD embedded between the metallic and superconducting electrodes and side-coupled to the topological nanowire with the edge Majorana quasiparticles (Fig. 1), can be described by the Anderson-type Hamiltonian

$$
H=H_{b a t h}+\sum_{\beta=S, N} H_{T, \beta}+H_{Q D}+H_{M Q D} .
$$

The bath $H_{\text {bath }}=H_{N}+H_{S}$ consists of the metallic $H_{N}=\sum_{k, \sigma} \xi_{k N} C_{k \sigma N}^{\dagger} C_{k \sigma N}$ and superconducting $H_{S}=$ $\sum_{k, \sigma} \xi_{k S} C_{k \sigma S}^{\dagger} C_{k \sigma S}-\sum_{k}\left(\Delta C_{k \uparrow S}^{\dagger} C_{-k \downarrow S}^{\dagger}+\right.$ h.c. $)$ reservoirs, where electron energies $\xi_{k \beta}$ are measured from the chemical potentials $\mu_{\beta}$. The central QD is described by $H_{Q D}=\sum_{\sigma} \epsilon d_{\sigma}^{\dagger} d_{\sigma}+U n_{\downarrow} n_{\uparrow}$, where $\epsilon$ denotes the energy level and $U$ stands for the repulsive interaction between opposite spin electrons. QD is hybridized with the external reservoirs by $H_{T, \beta}=\sum_{k, \sigma}\left(V_{k \beta} d_{\sigma}^{\dagger} C_{k \sigma \beta}+\right.$ h.c. $)$, where $V_{k \beta}$ denote the matrix elements.

Focusing on a subgap regime (i.e. energies $|\omega| \ll \Delta$ ) it has been shown ${ }^{61-63}$ that the superconducting electrode induces the static pairing. Its role can be thus played by the proximized quantum dot $H_{\text {prox }}=\sum_{\sigma} \epsilon d_{\sigma}^{\dagger} d_{\sigma}+$ $U n_{\downarrow} n_{\uparrow}-\frac{\Gamma_{S}}{2}\left(d_{\uparrow} d_{\downarrow}+d_{\downarrow}^{\dagger} d_{\uparrow}^{\dagger}\right)$. This simplification is fairly acceptable for our considerations of the spin-dependent subgap spectrum and the Andreev spectroscopy. Lowenergy theory of the Rashba nanowire can be expressed by 30

$$
H_{M Q D}=i \epsilon_{m} \eta_{1} \eta_{2}+\lambda\left(d_{\uparrow} \eta_{1}+\eta_{1} d_{\uparrow}^{\dagger}\right),
$$

where the operators $\eta_{i}=\eta_{i}^{\dagger}$ describe the edge states and $\epsilon_{m}$ accounts for their overlap. It is convenient to represent the exotic Majorana operators $\eta_{1}, \eta_{2}$ by the standard fermionic ones $\frac{8}{-} \eta_{1}=\frac{1}{\sqrt{2}}\left(f+f^{\dagger}\right), \eta_{2}=\frac{-i}{\sqrt{2}}\left(f+f^{\dagger}\right)$. In this representation the term (2) takes the following form

$$
H_{M Q D}=t_{m}\left(d_{\uparrow}^{\dagger}-d_{\uparrow}\right)\left(f+f^{\dagger}\right)+\epsilon_{m}\left(f^{\dagger} f+\frac{1}{2}\right),
$$

where $t_{m}=\lambda / \sqrt{2}$.

\section{B. Scattering on Majorana quasiparticles}

Let us denote the particle and hole Green's functions of the central QD coupled to the metallic and superconducting leads in absence of the Majorana quasiparticle by $\left\langle\left\langle d_{\sigma} ; d_{\sigma}^{\dagger}\right\rangle\right\rangle \equiv a^{-1}$ and $\left\langle\left\langle d_{\bar{\sigma}}^{\dagger} ; d_{\bar{\sigma}}\right\rangle\right\rangle \equiv b^{-1}$. For the uncorrelated case $(U=0)$ these functions read (B2)

$$
\begin{aligned}
& a=\omega-\epsilon+i \frac{\Gamma_{N}}{2}-\frac{\left(\Gamma_{S} / 2\right)^{2}}{\omega+\epsilon+i \frac{\Gamma_{N}}{2}}, \\
& b=\omega+\epsilon+i \frac{\Gamma_{N}}{2}-\frac{\left(\Gamma_{S} / 2\right)^{2}}{\omega-\epsilon+i \frac{\Gamma_{N}}{2}} .
\end{aligned}
$$


Similarly, we denote the inverse particle and hole propagators of the isolated Majorana quasiparticle by $m \equiv$ $\left(\omega-\epsilon_{m}\right)$ and $n \equiv\left(\omega+\epsilon_{m}\right)$, respectively.

Using the equation of motion approach (see Ap- pendix A) we calculated the matrix Green's function $\mathcal{G}_{\sigma}(\omega)=\left\langle\left\langle\Psi_{\sigma} ; \Psi_{\sigma}^{\dagger}\right\rangle\right\rangle$ defined in the matrix notation $\Psi_{\sigma}=$ $\left(d_{\sigma}, d_{\bar{\sigma}}^{\dagger}, f, f^{\dagger}\right)$. For spin $\uparrow$ the Green's function reads

$$
\mathcal{G}_{\uparrow}(\omega)=\frac{1}{W}\left(\begin{array}{cccc}
b m n-2 t_{m}^{2} \omega & -D\left(b m n-2 t_{m}^{2} \omega\right) & b n t_{m} & b m t_{m} \\
-D\left(b m n-2 t_{m}^{2} \omega\right) & \frac{1}{\omega-\epsilon+i \Gamma_{N} / 2}+D^{2}\left(b m n-2 t_{m}^{2} \omega\right) & -D b m t_{m} & -D b n t_{m} \\
b n t_{m} & -D b m t_{m} & a b n-(a+b) t_{m}^{2} & t_{m}^{2}(a+b) \\
b m t_{m} & -D b n t_{m} & (a+b) t_{m}^{2} & a b m-t_{m}^{2}(a+b)
\end{array}\right)
$$

where $W \equiv\left[a b m n-2 t_{m}^{2} \omega(a+b)\right]$ and $D \equiv\left(\Gamma_{S} / 2\right) /(\omega+$ $\left.\epsilon+i \Gamma_{N} / 2\right)$. In the same way, we also determined the matrix Green's function $\mathcal{G}_{\downarrow}(\omega)$. Below we present explicitly $\mathcal{G}_{\downarrow}^{(11)}(\omega)=\left\langle\left\langle d_{\downarrow} ; d_{\downarrow}^{\dagger}\right\rangle\right\rangle$ which yields the spectral function of $\downarrow$ electrons. It takes the following form

$$
\mathcal{G}_{\downarrow}^{(11)}(\omega)=G_{N}(\omega)+\left[\frac{\Gamma_{S}}{2} G_{N}(\omega)\right]^{2} \frac{a m n-2 t_{m}^{2} \omega}{a b m n-2 t_{m}^{2} \omega(a+b)} .(7)
$$

$G_{N}(\omega)=\left\langle\left\langle d_{\sigma} ; d_{\sigma}^{\dagger}\right\rangle\right\rangle$ is the Green's function for the case when QD is coupled only to the metallic lead (i.e. for $\left.\Gamma_{S}=0=t_{m}\right)$.

Let us remark that differences between $\mathcal{G}_{\uparrow}(\omega)$ and $\mathcal{G}_{\downarrow}(\omega)$ originate from the fact that only spin $\uparrow$ electrons are directly coupled to the Majorana mode. This difference vanishes for $t_{m} \rightarrow 0$ when $\mathcal{G}_{\sigma}^{11}$ reproduce the result $\underline{63}$ obtained for dot coupled only to $\mathrm{N}$ and $\mathrm{S}$ electrodes $\lim _{t_{m} \rightarrow 0} \mathcal{G}_{\downarrow}^{11}=\mathcal{G}_{\uparrow}^{11}=\left[\omega-\epsilon+i \Gamma_{N} / 2-\frac{\left(\Gamma_{S} / 2\right)^{2}}{\omega+\epsilon+i \Gamma_{N} / 2}\right]^{-1}$. On the other hand, for $t_{m} \neq 0$ in absence of a superconducting electrode $\left(\Gamma_{S}=0\right)$ the solution for spin $\downarrow$ electrons is identical with solution for QD coupled only to normal metal, regardless of the coupling strength to TS wire. This is because spin $\downarrow$ electrons are not affected by the side-coupled Majorana quasiparticle (so without electron pairing they do not 'feel' any interference). This result clearly indicates that the interference patterns appearing in the spectrum of $\downarrow$ electrons originate solely from the pairing with electrons of the opposite spin.

\section{Deviation from usual Fano shape}

In Fig. 22 we illustrate the spectral function $\rho_{\sigma}(\omega)$ of the central QD weakly coupled to the Majorana quasiparticle, in the case $\epsilon_{m}=0$. Interference pattern appearing at $\omega=0$ in the spectral function $\rho_{\uparrow}(\omega)$ resembles a resonant lineshape. However, from a careful examination we clearly notice that it is not really the true Fano resonance (like the one for $\uparrow$ electrons shown in Fig. 10). Such line-shape indicates, that electron waves resonantly scattered by the Majorana quasiparticle (to be regarded as half of the physical electron) change their phase only by the fraction of $\pi$ (which is typical value for scattering

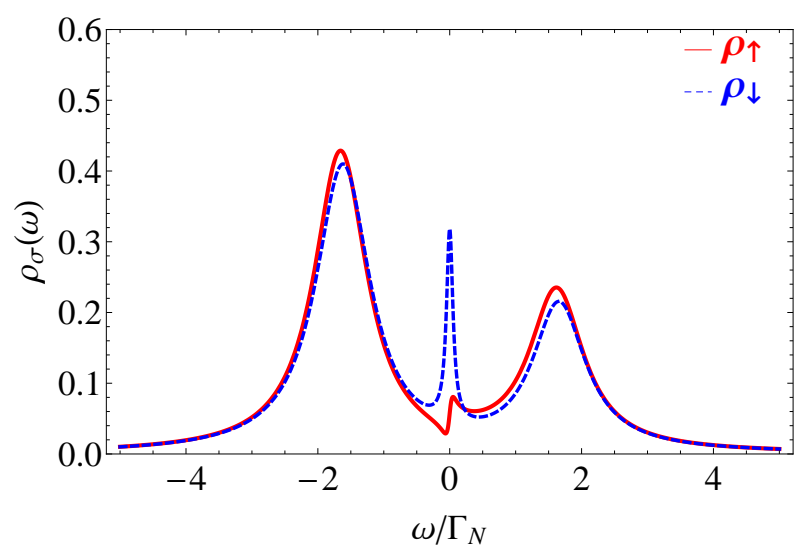

FIG. 2: Spectral function $\rho_{\sigma}(\omega)$ of the central dot coupled to MQD for $\epsilon_{m}=0$ using the model parameters $\Gamma_{S}=3 \Gamma_{N}$, $\epsilon=-0.5 \Gamma_{N}, t_{m}=0.3 \Gamma_{N}$. We notice that the interference pattern (at $\omega=0$ ) for $\uparrow$ electrons is different from the usual Fano shape.

caused by the side-coupled ordinary quantum dots 60 ). Mechanism responsible for this fractional interferometric feature has the same origin as $4 \pi$-periodicity of the Josephson junctions made of two 'majoranized' super-

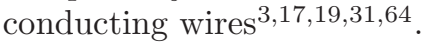

In Rashba nanowires of a finite length the Majorana quasiparticles partly overlap with one another, inducing some energy splitting between the edge modes $\left(\epsilon_{m} \neq 0\right)$. In Fig. 3 we show the interference patterns obtained for $\epsilon_{m}=\Gamma_{N}$. Spectrum of the spin $\uparrow$ electrons reveals two fractional Fano-like resonances (solid line in Fig. 31), whereas the spin $\downarrow$ electrons are characterized by two anti-Fano features (dashed lines in Fig. 3) at the same energies. Let us emphasize, that such behavior is qualitatively different from the results for T-shape heterojunction with the ordinary quantum dot (Fig. 10). Interferometric effects could thus be useful for detecting the Majorana quasiparticles in presence of electron pairing. 


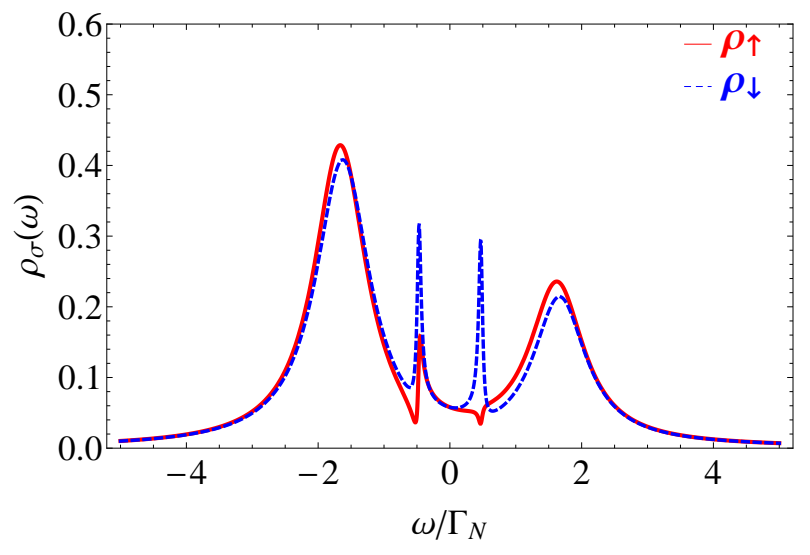

FIG. 3: Spectrum of the QD hybridized with the overlapping Majorana quasiparticles. Calculations have been done for $\epsilon_{m}=\Gamma_{N}$ using the same model parameters as in Fig. 2 .

\section{Evolution to 'molecular' region}

The interferometric Fano-like structures displayed in Figs 2 and 3 occur when the central QD is very weakly coupled to the side-attached Majorana quasiparticle. Upon increasing the interdot coupling $t_{m}$ the nanoscopic (QD and MQD) objects can be expected to develop some new spectroscopic signatures, characteristic for the entire 'molecular' complex.

Evolution of the spectral function $\rho_{\sigma}(\omega)$ vs $t_{m}$ is presented in Fig. 4. For increasing $t_{m}$ we observe that two Andreev peaks (originating from the mixed particle and hole degrees of freedom ${ }^{63}$ ) and the Fano/anti-Fano lineshapes (caused by the Majorana QD) gradually change into the three-peak structure. In both spin components we clearly see emergence of the zero-energy peak at expense of reducing the spectral weight of the initial Andreev states. Formation of the zero-energy peak signifies a 'leakage' of the Majorana quasiparticle into the central QD, in analogy to what has been discussed in Ref.55. In the present case such proximity induced zero-energy state affects both spin sectors, despite the fact that MQD is directly coupled only to the spin $\uparrow$ electrons.

\section{E. Majorana fingerprints in Andreev spectroscopy}

Interference effects caused by the Majorana quasiparticle can be practically observed in our setup (Fig. 1) by measuring the tunneling current under nonequilibrium conditions $\mu_{N} \neq \mu_{S}$. When applied voltage $\mu_{N}-\mu_{S} \equiv$ $\mathrm{eV}$ is smaller in magnitude than $\Delta$ the charge current $I_{A}(V)=\sum_{i} I_{A i}(V)$ is contributed by $\operatorname{spin} \uparrow(i \equiv 1)$ and spin $\downarrow(i \equiv 2)$ electrons. The spin-dependent Andreev currents can be expressed in Landauer form

$$
I_{A i}(V)=\frac{e}{h} \int d \omega T_{A i}(\omega)[f(\omega-e V)-f(\omega+e V)]
$$
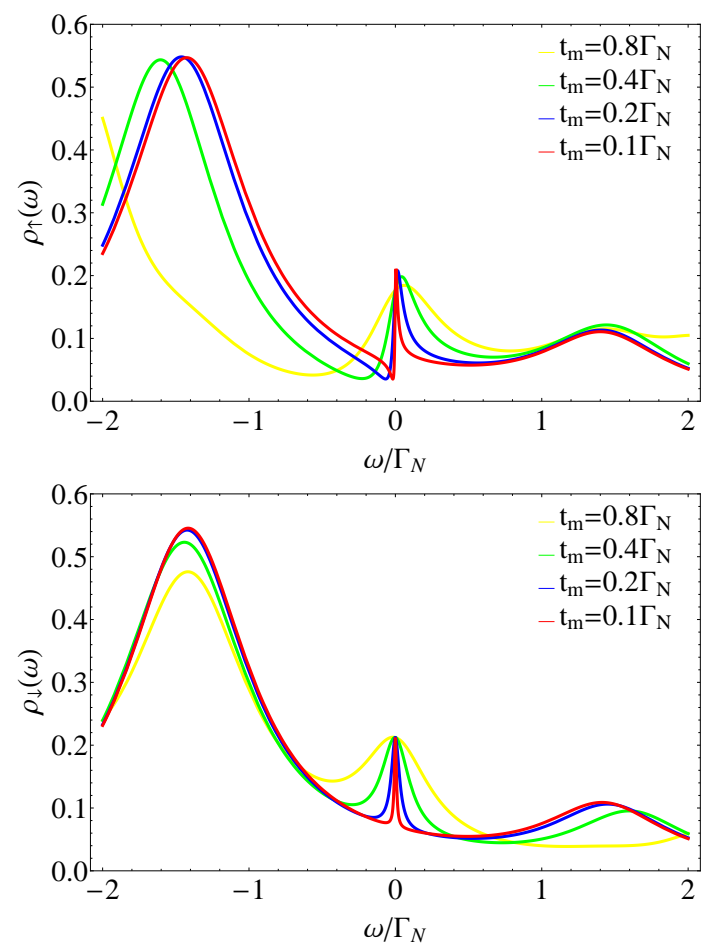

FIG. 4: Spectral function $\rho_{\sigma}(\omega)$ of the central QD obtained for $\sigma=\uparrow$ (upper panel) and $\sigma=\downarrow$ (lower panel) electrons, using $\Gamma_{S}=3 \Gamma_{N}, \epsilon_{m}=0$ and various couplings $t_{m}$, as indicated.

where $f(x)=\left[1+\exp \left(x / k_{B} T\right)\right]^{-1}$ is the Fermi distribution and transmittance for each spin sector

$$
T_{A i}(\omega)=\Gamma_{N}^{2} \times \begin{cases}\left|\left\langle\left\langle d_{\uparrow} ; d_{\downarrow}\right\rangle\right\rangle\right|^{2} & \text { for } i=1 \\ \left|\left\langle\left\langle d_{\downarrow} ; d_{\uparrow}\right\rangle\right\rangle\right|^{2} & \text { for } i=2\end{cases}
$$

describes a probability of converting the electron with spin $\sigma$ into the hole with spin $\bar{\sigma}$ in the metallic lead. The differential conductance $G_{A}(V)=d I_{A}(V) / d V$ is enhanced near the subgap (Andreev/Shiba) states ${ }^{63}$, but it is also sensitive to any other subgap features, including the quantum interference effects $\frac{47}{}$.

Fig. [5 shows the spin-resolved Andreev transmittance $T_{A i}(\omega)$ obtained for $\epsilon_{m}=0$ (upper panel) and $\epsilon_{m}=\Gamma_{N}$ (bottom panel). In the first case we observe the fractional resonance appearing for each spin sector at zerobias (although of opposite shapes). In the case $\epsilon_{m} \neq 0$ we notice two interferometric structures at $e V= \pm \epsilon_{m}$. For spin $\uparrow$ sector there appears the pronounced resonance at $e V=\epsilon_{m}$ and another shallow structure at $e V=-\epsilon_{m}$. The Andreev transmittance of $\downarrow$ sector has an opposite shape, i.e. $T_{A 2}(\omega)=T_{A 1}(-\omega)$. The spin-resolved Andreev transport could thus estimate the overlap $\epsilon_{m}$ between the edge modes of TS nanowire. For $\epsilon_{m}=0$ such spectroscopy can distinguish the fractional interferometric lineshapes caused by the Majorana quasiparticle from the typical Fano/antiFano lineshapes due to the normal quantum dots (see Appendix B). 

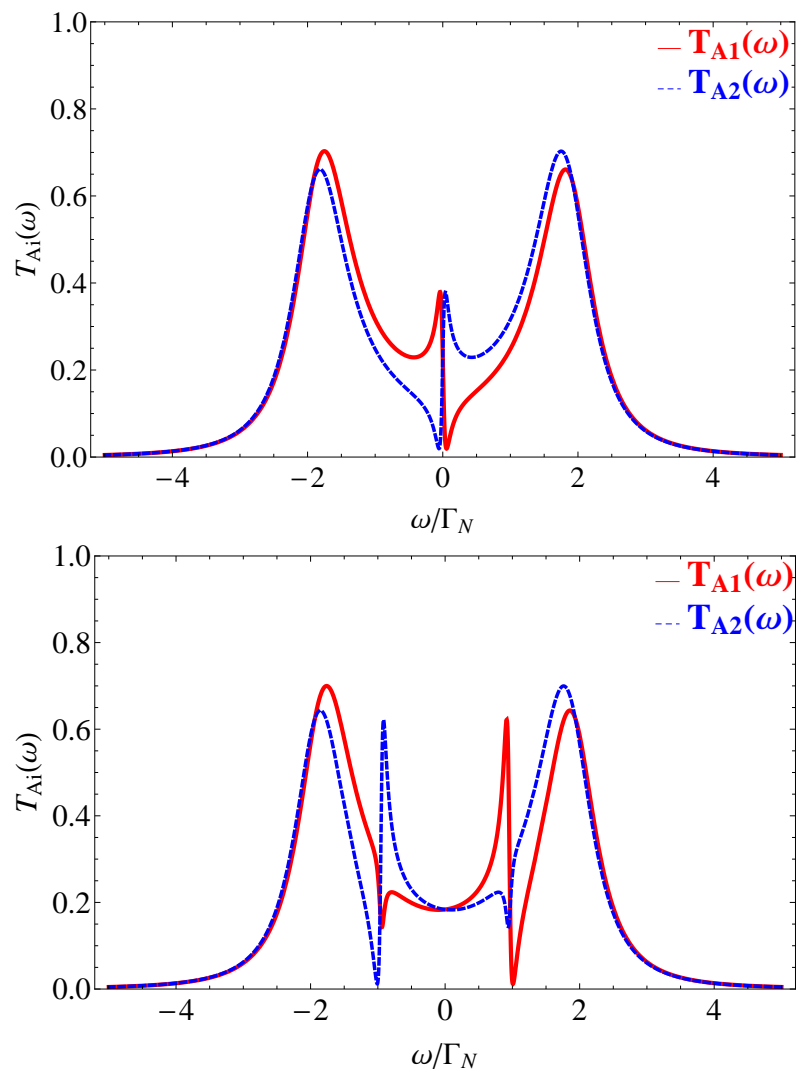

FIG. 5: The spin-resolved Andreev transmittance of the quantum dot strongly coupled to the superconducting lead $\Gamma_{S}=4 \Gamma_{N}$ and weakly hybridized with the side-attached Majorana quasiparticle $t_{m}=0.3 \Gamma_{N}$, where $\epsilon_{m}=0$ (upper panel) and $\epsilon_{m}=\Gamma_{N}$ (bottom panel). The red line refers to $\uparrow$ and the blue one to $\downarrow$ spin sectors.

\section{CORRELATION EFFECTS}

Since the interferometric feature caused by the sideattached Majorana quasiparticle shows up at the Fermi level (for the case $\epsilon_{m}=0$ ) it is natural to inspect its relationship with the Kondo effect, whose signature (narrow peak) appears at the same energy. The many-body Kondo effect occurs at low temperatures due to the effective exchange interaction induced between the QD and normal lead $(\mathrm{N})$ electrons. Its subtle nature in a subgap regime has been addressed by variety of methods (see the recent discussion 26,27 and other references cited therein).

\section{A. Methodological details}

In the present context we shall treat the correlations using the decoupling scheme for the Green's functions 47 that proved to be satisfactory on a qualitative level ${ }^{65}$. To account for the Kondo effect we start from the results obtained for the uncorrelated problem (section II) and proceed with approximations for the electron-electron interactions. In absence of the side-attached MQD, we again introduce the abbreviations for particle $\left\langle\left\langle d_{\sigma} ; d_{\sigma}^{\dagger}\right\rangle\right\rangle \equiv \tilde{a}^{-1}$ and hole $\left\langle\left\langle d_{\sigma}^{\dagger} ; d_{\sigma}\right\rangle\right\rangle \equiv \tilde{b}^{-1}$ propagators. Following our previous study ${ }^{63}$ of the single quantum dot (N-QD-S) setup we approximate these propagators by

$$
\begin{aligned}
& \tilde{a}=\omega-\epsilon-\Sigma_{N}(\omega)+\frac{\left(\Gamma_{S} / 2\right)^{2}}{\omega+\epsilon+\left[\Sigma_{N}(-\omega)\right]^{*}}, \\
& \tilde{b}=\omega+\epsilon+\left[\Sigma_{N}(-\omega)\right]^{*}+\frac{\left(\Gamma_{S} / 2\right)^{2}}{\omega-\epsilon-\Sigma_{N}(\omega)},
\end{aligned}
$$

where the selfenergy $\Sigma_{N}(\omega)$ accounts for the coupling of QD to the normal lead, taking into account the interactions between electrons $U n_{\downarrow} n_{\uparrow}$.

We approximate $\Sigma_{N}(\omega)$ using the popular decoupling scheme for the Green's functions (discussed in Appendix B of Ref $\stackrel{47}{)}$ which yields

$$
\begin{aligned}
\tilde{G}_{N}(\omega) & \equiv \frac{1}{\omega-\epsilon-\Sigma_{N}(\omega)} \\
& =\frac{\left.\omega-\epsilon-U\left(1-\left\langle n_{\sigma}\right\rangle\right)-\Sigma_{3}(\omega)\right]}{[\omega-\epsilon]\left[\omega-\epsilon-U-\Sigma_{3}(\omega)\right]+i \frac{\Gamma_{N}}{2} U},
\end{aligned}
$$

where

$$
\Sigma_{3}(\omega)=\sum_{k}\left|V_{k N}\right|^{2}\left[\frac{f\left(\xi_{k N}\right)}{\omega-\xi_{k N}}+\frac{f\left(\xi_{k N}\right)}{\omega-U-2 \varepsilon+\xi_{k N}}\right] .
$$

Alternatively one can treat the correlation effects at the central quantum within more sophisticated methods 56 .

Substituting the inverse Green's functions (10, 11) with the selfenergy $\Sigma_{N}(\omega)$ to the matrix Greens function (6) we obtain

$$
\begin{aligned}
\mathcal{G}_{\uparrow}^{11}(\omega) & =\frac{\tilde{b} m n-2 t_{m}^{2} \omega}{\tilde{W}} \\
\mathcal{G}_{\downarrow}^{(11)}(\omega) & =\tilde{G}_{N}(\omega)+\left[\frac{\Gamma_{S}}{2} \tilde{G}_{N}(\omega)\right]^{2} \frac{\tilde{a} m n-2 t_{m}^{2} \omega}{\tilde{W}}
\end{aligned}
$$

where $\tilde{W}=\tilde{a} \tilde{b} m n-2 t_{m}^{2} \omega(\tilde{a}+\tilde{b})$.

In our setup the correlated quantum dot is connected to the superconducting reservoir, which (by proximity effect) induces the on-dot electron pairing. On the other hand the repulsive Coulomb interactions disfavor any double occupancy, suppressing the local pairs. Even though the pairing and correlations are strongly antagonised one can find some regime of the model parameters, for which the Kondo physics coexists with the ondot pairing 27 (the latter is necessary for activating the Andreev tunnelling that could probe the subgap states). This regime is particularly important if we want to confront the Kondo state with the interferometric structures due to side-attached Majorana quasiparticle.

Optimal conditions where the Kondo effect coexists with the on-dot pairing can be tuned by $\varepsilon$ (that controls QD occupancy) and the ratio between couplings to external the electrodes $\Gamma_{S} / \Gamma_{N}$ (that is crucial for the effective 
exchange potential27). For specific calculations we focus here on the strong Coulomb potential $U=25 \Gamma_{N}$ and choose $\epsilon=-2 \Gamma_{N}$. We have checked that in such situation the Kondo effect coexists with the on-dot pairing for slightly asymmetric couplings $\Gamma_{S} \in\left(2 \Gamma_{N}, 6 \Gamma_{N}\right)$. With this in mind, we thus fixed the ratio $\Gamma_{S} / \Gamma_{N}=4$. In absence of MQD (i.e. for N-QD-S configuration) the narrow Kondo peak at $\omega=0$ coexists then with the subgap Andreev quasiparticle peaks at $\omega \approx \pm \sqrt{\epsilon^{2}+\left(\Gamma_{S} / 2\right)^{2}}$ whose broadening (inverse life-time) is proportional to $\Gamma_{N} \underline{\underline{63}}$.

\section{B. Majorana vs Kondo feature}
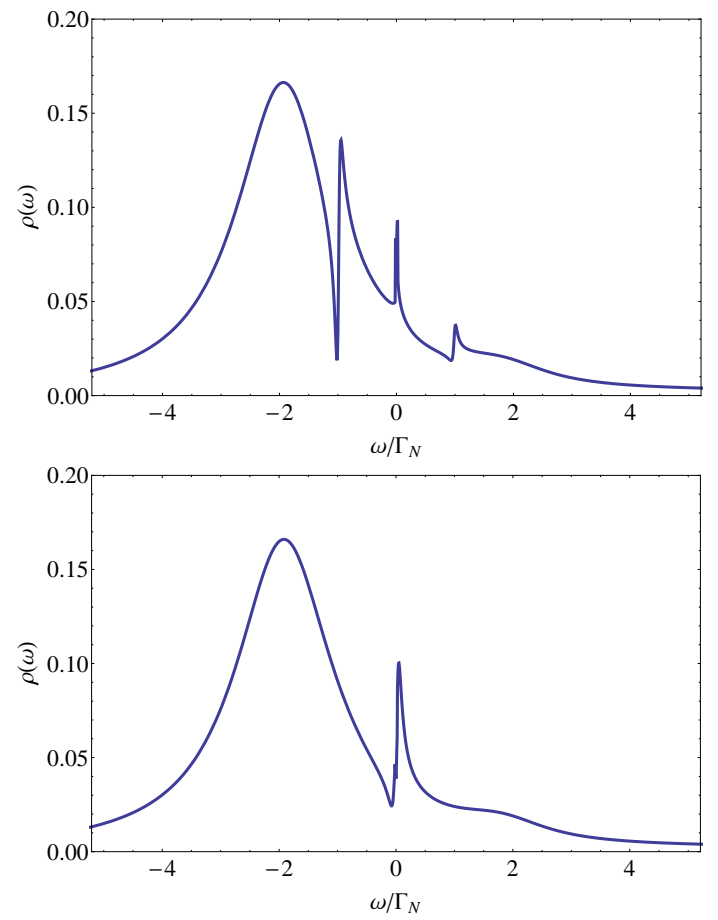

FIG. 6: Spectral function $\rho(\omega)$ of the correlated QD obtained in the Kondo regime for $\uparrow$ electrons using $\epsilon=-2 \Gamma_{N}, U=$ $25 \Gamma_{N}, \Gamma_{S}=4 \Gamma_{N}, t_{m}=0.3 \Gamma_{N}$. The upper panel refers to $\epsilon_{m}=\Gamma_{N}$ and the bottom one to $\epsilon_{m}=0$.

Influence of the side-coupled Majorana quasiparticle on the spin-resolved spectral functions $\rho_{\sigma}(\omega)$ of the correlated QD is illustrated in figures 6 and 7 . The upper panels correspond to the case of overlapping Majorana modes $\epsilon_{m}=\Gamma_{N}$. In analogy to the noninteracting situation (Fig. 3) we observe the fractional Fano and antiFano lineshapes appearing at $\omega= \pm \epsilon_{m}$ in the spectrum of spin $\uparrow$ and $\downarrow$ electrons, respectively. For $\downarrow$ electrons we also notice that both anti-Fano resonances are much less pronounced as compared to $U=0$ case. This is a consequence of the strong Coulomb interactions suppressing the on-dot pairing, that is indirectly responsible for the interferometric structures in the spectrum of $\downarrow$ electrons.

The most intriguing case occurs for $\epsilon_{m}=0$, when the
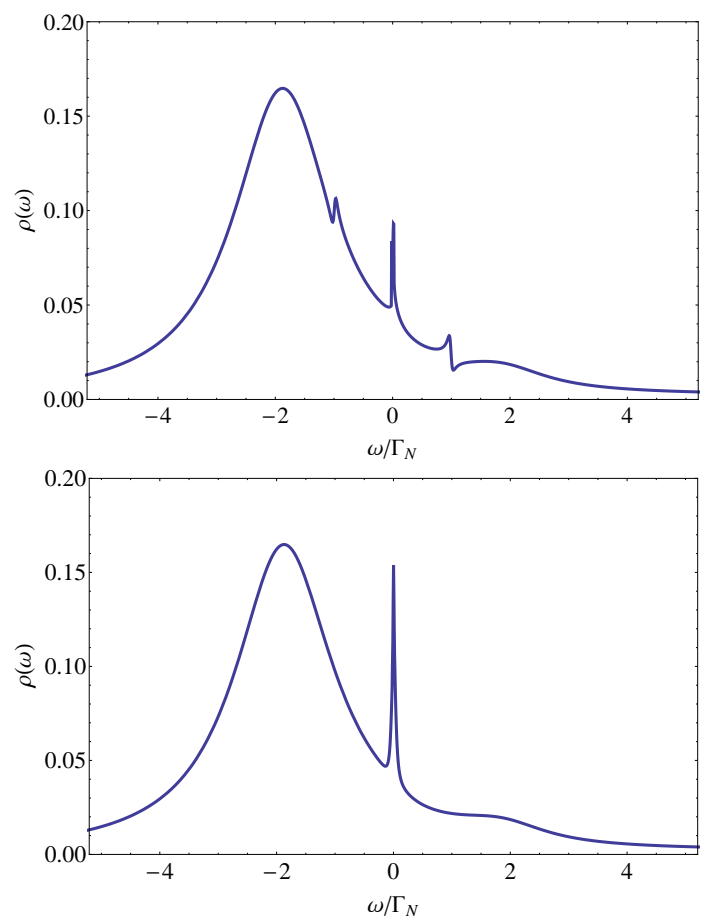

FIG. 7: Spectral function of the spin $\downarrow$ electrons obtained for the same set of model parameters as in figure 6 .

Kondo and interferometric structures coincide at exactly the same energy. Spectrum of $\uparrow$ electrons, that are directly coupled to the Majorana quasiparticle clearly show the dominant and destructive influence of the quantum interference on the Kondo state (see the bottom panel in Fig. 6, where the Kondo peak is completely washed out). As regards the spectrum of $\downarrow$ electrons, the anti-Fano interferometric structure constructively combines with the Kondo peak, enhancing the zero-energy feature.

Scattering mechanism driven by the zero-energy Majorana quasiparticle side-attached to the correlated quantum dot has thus very interesting effect on the Kondo state. For the spin $\uparrow$ sector (directly coupled to the Majorana quasiparticle) the ongoing quantum interference has destructive character. In other words, the fractional Fano-type resonance induced by the side-coupled Majorana quasiparticle is robust against the Kondo peak. On contrary, in the spin $\downarrow$ sector (where electrons are not directly coupled to the Majorana quasiparticle) the Kondo state is promoted by the quantum interference. Such exotic spin-resolved quantum interference effects might be useful for experimental detection of the Majorana quasiparticle. From a physical point of view, this spin-resolved screening effects of the correlated quantum dot is due to the following mechanism: the spin $\uparrow$ electrons 'leak' into the side-coupled Majorana structure (hence there is less spin $\uparrow$ to be screened), whereas the on-dot pairing compensates such loss by enhancing the density of $\downarrow$ electrons whose screening is effectively pronounced.

Practical observation of the Majorana and Kondo sig- 


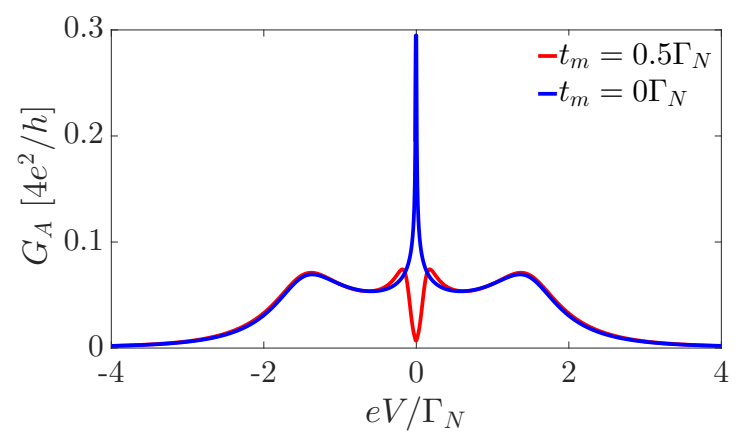

FIG. 8: Differential conductance $G_{A}$ of the subgap current (8) as a function of the applied voltage $V$ for $\epsilon=-1.5 \Gamma_{N}$, $U=25 \Gamma_{N}, \Gamma_{S}=4 \Gamma_{N}$ and $\epsilon_{m}=0$. The blue line corresponds to $t_{m}=0$ and the red curve is obtained for $t_{m}=0.5 \Gamma_{N}$.

natures would be feasible only indirectly, by measuring a differential conductance $G_{A}=d I_{A}(V) / d V$ of the net subgap current (8). Since both effects appear at zero energy, they shhuld be manifested in the linear conductance (i.e. at $V=0$ ). In absence of the side-attached Majorona quasiparticle (blue line in Fig. 8) we indeed observe a zero-bias enhancement driven by the subgap Kondo effect, that has been reported experimentally by several groups $66-69$. For the case, when Majorana quasiparticle is side-coupled to the interracial QD (red line in Fig. 8) there appears the dip at $V=0$ instead of the previous enhancement. Such destructive effect could thus distinguish between the Kondo and Majorana features. Furthermore, in realistic situation the magnetic field (which is necessary for inducing the zero-energy mode of TS wire) would additionally split the Kondo resonance, shifting it from $V=0$. In the configuration discussed here the zero-bias dip caused by the Majorana quasiparticle is hence robust against eventual spectroscopic feature of the Kondo effect.

\section{SUMMARY}

We studied interferometric structures induced by the Majorana quasiparticle side-coupled to the quantum dot on interface between the superconducting and normal electrodes. Due to the superconducting proximity effect such lineshapes appear simultaneously in both spin sectors, even though only one of the spins is directly coupled to the Majorana state. For each spin component, however, they are manifested differently.

The subgap spectrum of $\uparrow$ electrons (directly coupled to the Majorana quasiparticle) is characterized by the fractional Fano-type lineshapes. Their fractionality is caused by the fact that Majorana quasiparticle is half of a true electronic state. On the other hand, the spectrum of opposite spin electrons is characterized by anti-Fano lineshapes appearing at the same energies as for $\uparrow$ electrons. Such quantum interference does effectively yield different (spin-resolved) Andreev transmittances.

We also confronted the spin-resolved interferometric features with the Kondo effect (caused by the strong correlations). We found that the side-coupled Majorana quasiparticle can either suppress or enhance the Kondo effect, depending on the spin orientation. Screening of electrons that are directly coupled to the Majorana quasiparticle is practically destroyed by the quantum interference, whereas for the opposite spin component reveals substantial enhancement of the Kondo peak. We hope that our results can stimulate experimental efforts to verify the spin-selective influence of the Majorana quasiparticles on the Kondo state.

\section{Acknowledgments}

We acknowledge P. Stefański for valuable discussions and thank K.J. Kapcia for technical assistance. This work is supported by the National Science Centre in Poland via project DEC-2014/13/B/ST3/04451 (TD).

\section{Appendix A: Derivation of Green's functions}

In this appendix we outline procedure for determination of the matrix Green's function (6). Starting from the model Hamiltonian (1) we consider the uncorrelated quantum dot $U=0$ coupled to between the metallic $(\mathrm{N})$ and superconducting (S) electrodes and additionally side-coupled to the edge of topological wire. In the deep subgap regime the superconducting electrode induces the static on-dot pairing and (1) simplifies to

$$
\begin{aligned}
H & =H_{N}+H_{T, N}+\frac{\Gamma_{S}}{2}\left(d_{\uparrow}^{\dagger} d_{\downarrow}^{\dagger}+d_{\downarrow} d_{\uparrow}\right)+\sum_{\sigma} \epsilon d_{\sigma}^{\dagger} d_{\sigma} \\
& +t_{m}\left(d_{\uparrow}^{\dagger}-d_{\uparrow}\right)\left(f^{\dagger}+f\right)+\epsilon_{m}\left(f^{\dagger} f+1 / 2\right) .
\end{aligned}
$$

Fourier transform of the retarded Green's function can be computed from the equation of motion $\omega\langle\langle A ; B\rangle\rangle=$ $\left\langle[A, B]_{+}\right\rangle+\left\langle\left\langle[A, H]_{-} ; B\right\rangle\right\rangle$ where \pm denote anticommutator/commutator, respectively. The particle propagator $\left\langle\left\langle d_{\uparrow} ; d_{\uparrow}^{\dagger}\right\rangle\right\rangle$ for spin $\uparrow$ electrons of the central QD is mixed with the operators of TS wire and with the anomalous Green's function

$$
\begin{aligned}
&\left(\omega-\epsilon+i \frac{\Gamma_{N}}{2}\right)\left\langle\left\langle d_{\uparrow} ; d_{\uparrow}^{\dagger}\right\rangle\right\rangle=1-\frac{\Gamma_{S}}{2}\left\langle\left\langle d_{\downarrow}^{\dagger} ; d_{\uparrow}^{\dagger}\right\rangle\right\rangle \\
&+t_{m}\left\langle\left\langle\left(f^{\dagger}+f\right) ; d_{\uparrow}^{\dagger}\right\rangle\right\rangle
\end{aligned}
$$

As $\downarrow$ electrons are not directly coupled to TS wire the anomalous Green's function $\left\langle\left\langle d_{\downarrow}^{\dagger} ; d_{\uparrow}^{\dagger}\right\rangle\right\rangle$ does not generate any propagator containing $f$ operators. Using EOM, it can be expressed via the hole propagator

$$
\left(\omega+\epsilon+i \frac{\Gamma_{N}}{2}\right)\left\langle\left\langle d_{\downarrow}^{\dagger} ; d_{\uparrow}^{\dagger}\right\rangle\right\rangle=-\frac{\Gamma_{S}}{2}\left\langle\left\langle d_{\uparrow} ; d_{\uparrow}^{\dagger}\right\rangle\right\rangle
$$


Using (A3) we can rewrite equation (A2) as

$$
\begin{array}{r}
\left(\omega-\epsilon+i \frac{\Gamma_{N}}{2}-\frac{\left(\Gamma_{S} / 2\right)^{2}}{\omega+\epsilon+i \Gamma_{N} / 2}\right)\left\langle\left\langle d_{\uparrow} ; d_{\uparrow}^{\dagger}\right\rangle\right\rangle= \\
1+t_{m}\left\langle\left\langle f^{\dagger} ; d_{\uparrow}^{\dagger}\right\rangle\right\rangle+t_{m}\left\langle\left\langle f ; d_{\uparrow}^{\dagger}\right\rangle\right\rangle .
\end{array}
$$

We can notice that expression in the bracket on left hand side is the inverse particle propagator for N-QD-S system in absence of the TS wire. For brevity we denote it by symbol $a$, that is presented in Eqn (4).

The other Green's functions, where $d_{\sigma}^{\dagger}$ is mixed with $f$ and $f^{\dagger}$ operators can be found from the EOM as

$$
\begin{aligned}
&\left(\omega-\epsilon_{m}\right)\left\langle\left\langle f ; d_{\uparrow}^{\dagger}\right\rangle\right\rangle=t_{m}\left\langle\left\langle d_{\uparrow} ; d_{\uparrow}^{\dagger}\right\rangle\right\rangle-t_{m}\left\langle\left\langle d_{\uparrow}^{\dagger} ; d_{\uparrow}^{\dagger}\right\rangle\right\rangle( \\
&\left(\omega+\epsilon_{m}\right)\left\langle\left\langle f^{\dagger} ; d_{\uparrow}^{\dagger}\right\rangle\right\rangle=t_{m}\left\langle\left\langle d_{\uparrow} ; d_{\uparrow}^{\dagger}\right\rangle\right\rangle-t_{m}\left\langle\left\langle d_{\uparrow}^{\dagger} ; d_{\uparrow}^{\dagger}\right\rangle\right\rangle(
\end{aligned}
$$

Equations A5|A6 generate the new anomalous function $\left\langle\left\langle d_{\uparrow}^{\dagger} d_{\uparrow}^{\dagger}\right\rangle\right\rangle$. We write down the equation of motion for this function

$$
\begin{array}{r}
\left(\omega+\epsilon+i \frac{\Gamma_{N}}{2}\right)\left\langle\left\langle d_{\uparrow}^{\dagger} ; d_{\uparrow}^{\dagger}\right\rangle\right\rangle=\frac{\Gamma_{S}}{2}\left\langle\left\langle d_{\downarrow} ; d_{\uparrow}^{\dagger}\right\rangle\right\rangle \\
-t_{m}\left(\left\langle\left\langle f^{\dagger} ; d_{\uparrow}^{\dagger}\right\rangle\right\rangle+\left\langle\left\langle f ; d_{\uparrow}^{\dagger}\right\rangle\right\rangle\right)
\end{array}
$$

and determine the new function $\left\langle\left\langle d_{\downarrow} ; d_{\uparrow}^{\dagger}\right\rangle\right\rangle$ as

$$
\left(\omega-\epsilon+i \frac{\Gamma_{N}}{2}\right)\left\langle\left\langle d_{\downarrow} ; d_{\uparrow}^{\dagger}\right\rangle\right\rangle=\frac{\Gamma_{S}}{2}\left\langle\left\langle d_{\uparrow}^{\dagger} ; d_{\uparrow}^{\dagger}\right\rangle\right\rangle .
$$

Now the propagator $\left\langle\left\langle d_{\uparrow}^{\dagger} ; d_{\uparrow}^{\dagger}\right\rangle\right\rangle$ can be represented as

$$
\begin{array}{r}
\left(\omega+\epsilon+i \Gamma_{N} / 2-\frac{\left(\Gamma_{S} / 2\right)^{2}}{\omega-\epsilon+i \Gamma_{N} / 2}\right)\left\langle\left\langle d_{\uparrow} ; d_{\uparrow}^{\dagger}\right\rangle\right\rangle= \\
-t_{m}\left(\left\langle\left\langle f^{\dagger} ; d_{\uparrow}^{\dagger}\right\rangle\right\rangle+\left\langle\left\langle f ; d_{\uparrow}^{\dagger}\right\rangle\right\rangle\right) .
\end{array}
$$

Expression appearing in a bracket on the left hand side is the inverse hole propagator of N-QD-S system without TS wire. We have denoted it by symbol $b$ in the main text, that is explicitly given by Eqn (5).

Finally, we obtain the following set of equations

$$
\begin{aligned}
a\left\langle\left\langle d_{\uparrow} ; d_{\uparrow}^{\dagger}\right\rangle\right\rangle & =1+t_{m}\left\langle\left\langle f^{\dagger} ; d_{\uparrow}^{\dagger}\right\rangle\right\rangle+t_{m}\left\langle\left\langle f ; d_{\uparrow}^{\dagger}\right\rangle\right\rangle, \\
b\left\langle\left\langle d_{\uparrow}^{\dagger} ; d_{\uparrow}^{\dagger}\right\rangle\right\rangle & =-t_{m}\left\langle\left\langle f^{\dagger} ; d_{\uparrow}^{\dagger}\right\rangle\right\rangle-t_{m}\left\langle\left\langle f ; d_{\uparrow}^{\dagger}\right\rangle,\right. \\
\left(\omega-\epsilon_{m}\right)\left\langle\left\langle f ; d_{\uparrow}^{\dagger}\right\rangle\right\rangle & =t_{m}\left\langle\left\langle d_{\uparrow} ; d_{\uparrow}^{\dagger}\right\rangle\right\rangle-t_{m}\left\langle\left\langle d_{\uparrow}^{\dagger} ; d_{\uparrow}^{\dagger}\right\rangle\right\rangle, \\
\left(\omega+\epsilon_{m}\right)\left\langle\left\langle f^{\dagger} ; d_{\uparrow}^{\dagger}\right\rangle\right\rangle & =t_{m}\left\langle\left\langle d_{\uparrow} ; d_{\uparrow}^{\dagger}\right\rangle\right\rangle-t_{m}\left\langle\left\langle d_{\uparrow}^{\dagger} ; d_{\uparrow}^{\dagger}\right\rangle\right\rangle .
\end{aligned}
$$

Using the abbreviations $m \equiv\left(\omega-\epsilon_{m}\right), n \equiv\left(\omega+\epsilon_{m}\right)$ and denoting $W \equiv a b m n-2 t_{m}^{2} \omega(a+b)$ these Green's functions can be recast in the following matrix form

$$
\left(\begin{array}{cc}
\left\langle\left\langle d_{\uparrow} ; d_{\uparrow}^{\dagger}\right\rangle\right\rangle & \left\langle\left\langle f ; d_{\uparrow}^{\dagger}\right\rangle\right\rangle \\
\left\langle\left\langle f^{\dagger} ; d_{\uparrow}^{\dagger}\right\rangle\right\rangle & \left\langle\left\langle d_{\uparrow}^{\dagger} ; d_{\uparrow}^{\dagger}\right\rangle\right\rangle
\end{array}\right)=\frac{1}{W}\left(\begin{array}{cc}
b m n-2 t_{m}^{2} \omega & b n t_{m} \\
b m t_{m} & -2 t_{m}^{2} \omega
\end{array}\right)
$$

The entire matrix presented in Eqn (6) can be obtained by solving 4 similar sets of the equations.

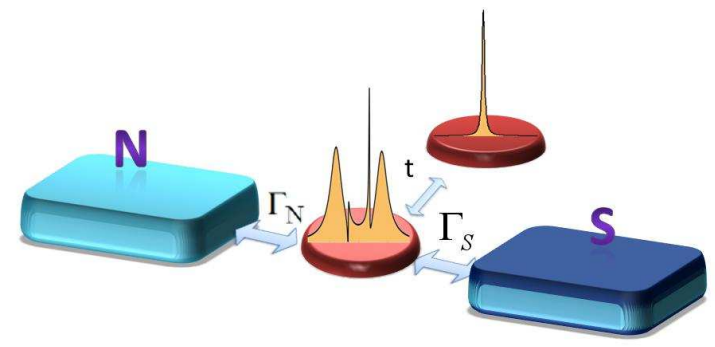

FIG. 9: Schematic view of the Fano and anti-Fano interference patterns induced in the T-shape setup with both normal quantum dots for $t_{\uparrow}=t_{\downarrow}$.

\section{Appendix B: Spin-dependent coupling to normal QD}

To distinguish the consequences caused by the fact that tunneling to the MQD involves only the spin $\uparrow$ electrons from other effects due to their specific Majorana-type nature we examine here the setup in which TS is replaced by the usual quantum $\operatorname{dot}\left(\mathrm{QD}_{2}\right)$

$$
H_{M Q D} \rightarrow H_{Q D_{2}}=\sum_{\sigma} \epsilon_{2} d_{2 \sigma}^{\dagger} d_{2 \sigma}+\sum_{\sigma} t_{\sigma}\left(d_{\sigma}^{\dagger} d_{2 \sigma}+\text { h.c. }\right) .
$$

Our previous study 47 of such normal double quantum dot (DQD) in the T-shape configuration has been done for the spin independent couplings $t_{\uparrow}=t_{\downarrow}$. Under such circumstances we have obtained the Fano and anti-Fano resonances, schematically displayed in Fig. 9.

In this Appendix we address the spin-polarized coupling $t_{\uparrow} \neq t_{\downarrow}$, focusing on the limit of vanishing $t_{\downarrow}$. Fourier transform of the retarded Green's function for the uncorrelated central quantum dot is expressed by $\underline{47}$

$$
\begin{aligned}
& {\left[\begin{array}{cc}
\left\langle\left\langle d_{\sigma} ; d_{\sigma}^{\dagger}\right\rangle\right\rangle & \left\langle\left\langle d_{\sigma} ; d_{\bar{\sigma}}\right\rangle\right\rangle \\
\left\langle\left\langle d_{\bar{\sigma}}^{\dagger} ; d_{\sigma}^{\dagger}\right\rangle\right\rangle & \left\langle\left\langle d_{\bar{\sigma}}^{\dagger} ; d_{\bar{\sigma}}\right\rangle\right\rangle
\end{array}\right] } \\
= & \left(\begin{array}{cc}
\omega-\epsilon+\frac{i \Gamma_{N}}{2}-\frac{t_{\sigma}^{2}}{\omega-\epsilon_{2}} & \frac{\Gamma_{S}}{2} \\
\frac{\Gamma_{S}}{2} & \omega+\epsilon+\frac{i \Gamma_{N}}{2}-\frac{t_{\sigma}^{2}}{\omega+\epsilon_{2}}
\end{array}\right)^{-1}
\end{aligned}
$$

where $\bar{\sigma}$ is inverse spin to $\sigma$. For the weak identical couplings $t_{\uparrow}=t_{\downarrow}$ the spectral function of central quantum $\operatorname{dot} \rho_{\sigma}(\omega)=-\pi^{-1} \operatorname{Im}\left\langle\left\langle d_{\sigma} ; d_{\sigma}^{\dagger}\right\rangle\right\rangle$ is characterized by two interferometric structures at the $\mathrm{QD}_{2}$ level and on the opposite side of a Fermi level $\stackrel{47}{ }$. The feature at $\omega=\epsilon_{2}$ has the usual Fano-type resonant lineshape ${ }^{60}$, whereas its companion at $-\epsilon_{2}$ has anti-resonant (antiFano) shape. Obviously for $t_{\uparrow}=t_{\downarrow}$ the spectral functions $\rho_{\sigma}(\omega)$ of both spins are identical.

When the tunneling of $\downarrow$ electrons is forbidden $\left(t_{\downarrow}=0\right)$, the toy model (B1) is closely analogous to the original setup (Fig. 1) with only spin $\uparrow$ of the central quantum dot coupled to TS wire. In such case there survives the single interferometric structure in each of the spectral functions $\rho_{\sigma}(\omega)$. For $\uparrow$ electrons (directly coupled to the side-attached dot) we observe the Fano-type interference 

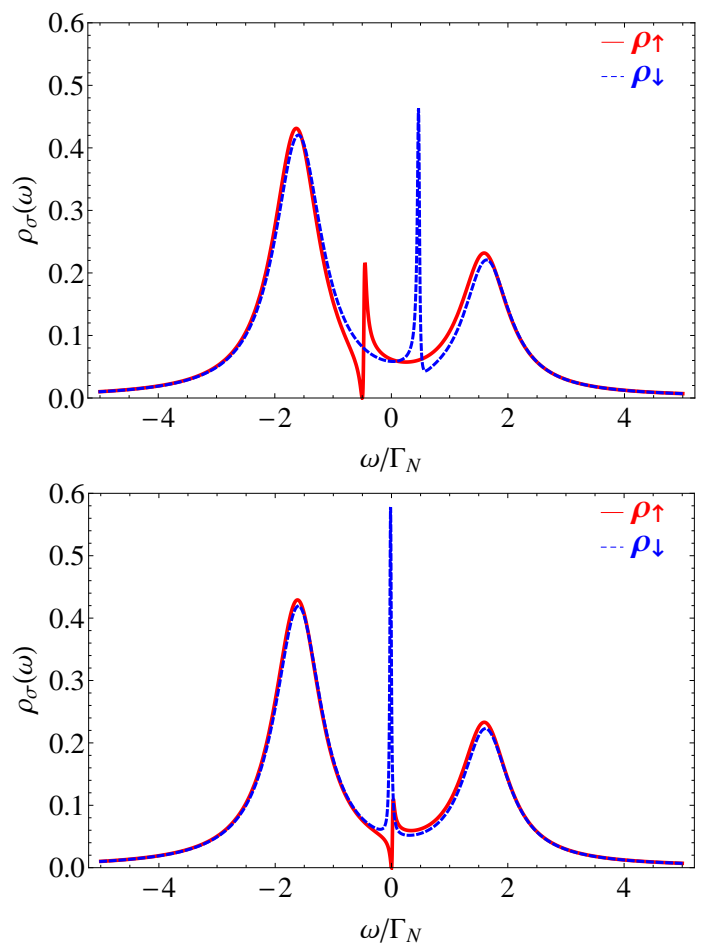

FIG. 10: Spectral function $\rho_{\sigma}(\omega)$ of the uncorrelated QD asymmetrically coupled $\left(t_{\uparrow}=0.3 \Gamma_{N}, t_{\downarrow}=0\right)$ to the normal $\mathrm{QD}_{2}$. The solid (red ) line refers to $\uparrow$ electrons and the dashed (blue) once to spin $\downarrow$ electrons. The results are obtained for the model parameters $\Gamma_{S}=3 \Gamma_{N}, \epsilon=-0.5 \Gamma_{N}$ and $\epsilon_{2}=0$. We notice the usual Fano-type pattern for $\uparrow$ electrons (directly coupled to QD) accompanied by the anti-Fano feedback for spin $\downarrow$ electrons (due to the on-dot pairing). Top panel refers to $\epsilon_{2}=-0.5 \Gamma_{N}$ and the bottom one to $\epsilon_{2}=0$.

pattern at $\varepsilon_{2}$ and for the opposite spin $\downarrow$ electrons there appears the anti-Fano structure at $-\varepsilon_{2}$. This result can be understood if we anticipate that the anti-Fano feature is an indirect response of the spin $\uparrow$ electrons. In other words, even though the spin $\downarrow$ electrons are not directly coupled to the side-attached quantum dot, due to the induced local pairing they 'feel' a feedback from the opposite $(\uparrow)$ spin electrons.

The upper panel in figure 10 illustrates the spinresolved spectral functions of the uncorrelated central quantum dot asymmetrically coupled to the normal $\mathrm{QD}_{2}$ whose energy level is $\epsilon_{2} \neq 0$, where interferometric features appear either in the particle or hole regions. The bottom panel in figure 10 corresponds to the situation with $\epsilon_{2}=0$. In this case the Fano and anti-Fano lineshapes appear at the same position what is partly similar to the case with Majorana quasiparticle.

Figure 11 shows the spectral function $\rho_{\sigma}(\omega)$ obtained fairly below the Kondo temperature $T_{K}$ for spin $\uparrow$ (red line) and $\downarrow$ (blue line) electrons. The approximation described in Sec. III.A cannot reliably reproduce the low energy structure of the Kondo peak $|\omega| \geq k_{B} T_{K}$, therefore our results should be treated only qualitatively.
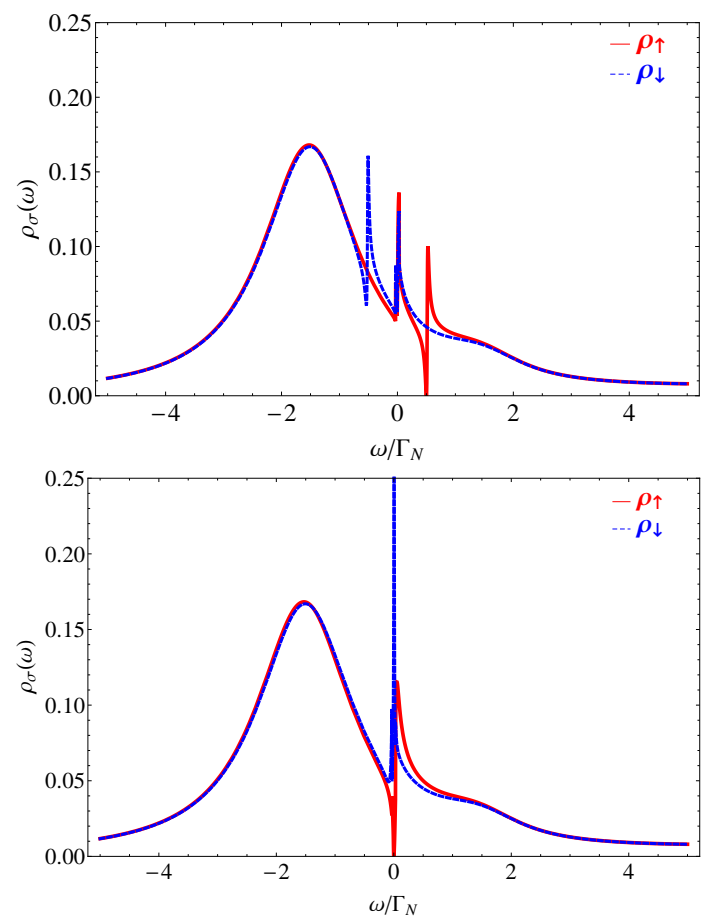

FIG. 11: Spectral function the correlated QD side-coupled to the normal $\mathrm{QD}_{2}$ obtained for $\Gamma_{S}=4 \Gamma_{N}, U=15 \Gamma_{N}, k_{B} T=$ $0.005 \Gamma_{N}$. The solid (blue) line refers to $\uparrow$ and the dashed (red) line to $\downarrow$ electrons. Top panel refers to $\epsilon_{2}=0.5 \Gamma_{N}$ and the bottom one to $\epsilon_{2}=0$.

In the case when $\epsilon_{2}$ is far from the Kondo peak (top panel) we observe that the Fano-type resonance (seen in $\rho_{\uparrow}(\omega)$ at $\left.\epsilon_{2}\right)$ and its anti-Fano companion (present in $\rho_{\downarrow}(\omega)$ at $\left.-\epsilon_{2}\right)$ practically coexist with the zero-energy Kondo peak. The situation changes dramatically, when energy $\epsilon_{2}$ coincides with the Fermi level (bottom panel). In both spin sectors the Kondo peak is then completely destroyed by the interferometric lineshape. This effect proves that the quantum interference is dominant, whenever it coincides with the Kondo peak. Let us notice that such tendency is distinct from the interferometric features induced by the Majorana quasiparticle (Figs 6 and (7).

\section{Appendix C: Influence of the trivially paired dot}

In analogy to Appendix B, we consider now the sideattached quantum dot $\mathrm{QD}_{2}$ characterized by the usual $s$ wave pairing. Physically such situation can be achieved in STM-type configuration illustrated by Fig. 12. The charge transport would occur between the normal tip $(\mathrm{N})$ and the superconducting lead (Sc) through the quantum dot $\left(\mathrm{QD}_{1}\right)$ deposided on superconductor and laterally coupled to another quantum dot $\left(\mathrm{QD}_{2}\right)$, that rests on the same superconducting substrate. This setup can be described by the model similar to (B1) with the $s$-wave pairing imposed on $\mathrm{QD}_{2}$. Formally we use the follow- 


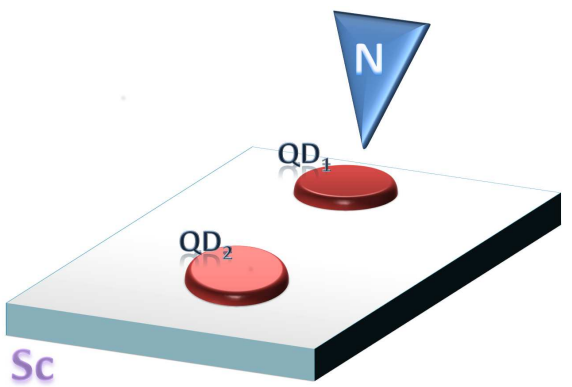

FIG. 12: Schematic view of STM-type configuration, where transport can occur between the normal tip (N) and the superconducting $(\mathrm{Sc})$ substrate via the central quantum dot $\left(\mathrm{QD}_{1}\right)$ which is laterally coupled to another quantum dot $\left(\mathrm{QD}_{2}\right)$. Both dots absorb the $s$-wave (trivial) pairing.

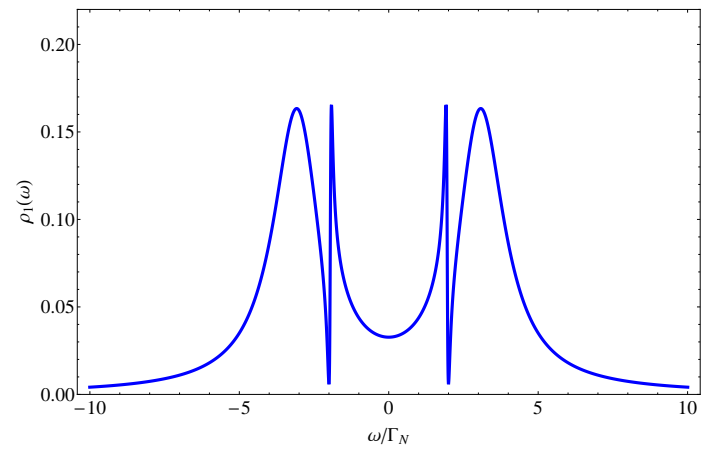

FIG. 13: Spectral function $\rho_{1}(\omega)$ of the interfacial quantum dot obtained for $\epsilon=0, \Gamma_{S}=6 \Gamma_{N}, \epsilon_{2}=0 \Gamma_{S 2}=4 \Gamma_{N}$, $t=0.3 \Gamma_{N}$, neglecting the correlations. ing model $H_{Q D_{2}}=\sum_{\sigma} \epsilon_{2} d_{2 \sigma}^{\dagger} d_{2 \sigma}-\left(\frac{\Gamma_{S 2}}{2} d_{2 \uparrow}^{\dagger} d_{2 \downarrow}^{\dagger}+\right.$ h.c. $)+$ $\sum_{\sigma}\left(t d_{1 \sigma}^{\dagger} d_{2 \sigma}+\right.$ h.c. $)$, where $\Gamma_{S 2}$ describes the effective coupling of $\mathrm{QD}_{2}$ to the $s$-wave superconducting reservoir.
Key difference between the TS wire (that hosts the Majorana mode) and the $\mathrm{QD}_{2}$ (that absorbs the usual $s$-wave superconductivity) can be observed in the electronic spectra. Majorana quasiparticle emerges at $\omega=0$, whereas the fermionic Shiba/Andreev states of $\mathrm{QD}_{2}$ are formed at finite energies $\omega= \pm \sqrt{\epsilon_{2}^{2}+\left(\Gamma_{S 2} / 2\right)^{2}}$.Influence of $\mathrm{QD}_{2}$ on the subgap spectrum of the central $\mathrm{QD}_{1}$ is visible away from the Fermi level. Figure 13 presents the spectral function $\rho_{1}(\omega)$ of $\mathrm{QD}_{1}$. As a matter of fact, its electronic spectrum qualitatively differs from the unique features due to Majorana quasiparticle, discussed in main part of this work (see Fig. 2).
* Electronic address: doman@kft.umcs.lublin.pl

1 Volovik G 1999 Fermion zero modes on vortices in chiral superconductors JETP Lett. 70609

${ }^{2}$ Read N and Green D 2000 Paired states of fermions in two dimensions with breaking of parity and time-reversal symmetries and the fractional quantum Hall effect Phys. Rev. B 6110267

3 Kitaev A Y 2001 Unpaired majorana fermions in quantum wires Phys. Usp. 44131

4 Alicea J 2012 New directions in the pursuit of Majorana fermions in solid state systems Rep. Prog. Phys. 75076501

${ }^{5}$ Leijnse M and Flensberg K 2012 Introduction to topological superconductivity and Majorana fermions Semicond. Sci. Technol. 27124003

6 Stanescu T D and Tewari S 2013 Majorana fermions in semiconductor nanowires: fundamentals, modeling, and experiment J. Phys.: Condens. Matter 25233201

7 Beenakker C W J 2013 Search for majorana fermions in superconductors Annu. Rev. Condens. Matt. Phys. 4113

8 Elliot S R and Franz M 2015 Colloquium: Majorana fermions in nuclear, particle, and solid-state physics Rev. Mod. Phys 87137

${ }^{9}$ Liu X, Li X, Deng D -L, Liu X -J and Das Sarma S 2016 Majorna spintronics Phys. Rev. B 94014511

10 Tewari S, Das Sarma S, Nayak C, Zhang C W and Zoller P
2007 Quantum computation using vortices and Majorana zero modes of a $p_{x}+i p_{y}$ superfluid of fermionic cold atoms Phys. Rev. Lett. 98010506

11 Fu L and Kane C L 2008 Superconducting proximity effect and Majorana fermions at the surface of a topological insulator Phys. Rev. Lett. 100096407

12 Nilsson J, Akhmerov A R and Beenakker C W J 2008 Splitting of a Cooper pair by a pair of Majorana bound state Phys. Rev. Lett. 101120403

13 Sato M and Fujimoto S 2009 Topological phases of noncentrosymmetric superconductors: edge states, Majorana fermions, and non-Abelian statistics Phys. Rev. B 79 094504

14 Wimmer M, Akhmerov A R, Medvedyeva M V, Tworzydło J and Beenakker C W J 2010 Majorana bound states without vortices in topological superconductors with electrostatic defects Phys. Rev. Lett. 105046803

15 Sau J D, Lutchyn R M, Tewari S and Das Sarma S 2010 Generic new platform for topological quantum computation using semiconductor heterostructures Phys. Rev. Lett. 104040502

16 Oreg Y, Refael G and von Oppen F 2010 Helical liquids and Majorana bound states in quantum wires Phys. Rev. Lett. 105177002

17 Lutchyn R M, Sau J D and Das Sarma S 2010 
Majorana fermions and a topological phase transition in semiconductor-superconductor heterostructures Phys. Rev. Lett. 105077001

18 Choy T P, Edge J M, Akhmerov A R and Beenakker C W J 2011 Majorana fermions emerging from magnetic nanoparticles on a superconductor without spin-orbit coupling Phys. Rev. B 84195442

19 San-Jose P, Prada E and Aguado R 2012 ac Josephson effect in finite-length nanowire junctions with Majorana modes Phys. Rev. Lett. 108257001

20 Jiang L, Kitagawa T, Alicea J, Akhmerov A R, Pekker D, Refael G, Cirac J I, Demler E, Lukin M D and Zoller P 2011 Majorana fermions in equilibrium and in driven cold-atom quantum wires Phys. Rev. Lett. 106220402

${ }^{21}$ Mourik V, Zuo K, Frolov S M, Plissard S R, Bakkers E P A M and Kouwenhoven L P 2012 Signatures of Majorana fermions in hybrid superconductor-semiconductor nanowire devices Science 3361003

22 Nadj-Perge S, Drozdov I K, Li J, Chen H, Jeon S, Seo J, MacDonald A H, Andrei Bernevig B and Yazdani A 2014 Observation of Majorana fermions in ferromagnetic atomic chains on a superconductor Science 346602

23 Pawlak R, Kisiel M, Klinovaja J, Maier T, Kawai S, Glatzel T, Loss D and Meyer E 2015 Probing atomic structure and Majorana wave-functions in mono-atomic Fe-chains on superconducting $\mathrm{Pb}$-surface (arXiv:1505.06078)

24 Ruby M, Pientka F, Peng Y, von Oppen F, Heinrich B W and Franke K J 2015 End states and subgap structure in proximity-coupled chains of magnetic adatoms Phys. Rev. Lett. 115197204

${ }^{25}$ Liu J, Potter A C, Law K T and Lee P A 2012 Zerobias in the tunneling conductance of spin-orbit-coupling superconducting wires with and without Majorana endstates Phys. Rev. Lett. 109267002

26 Žitko R, Lim J S, López R and Aguado R 2015 Shiba states and zero-bias anomalies in the hybrid normalsuperconductor Anderson model Phys. Rev. B 91045441

27 Domański T, Weymann I, Barańska M and Górski G 2016 Constructive influence of the induced electron pairing on the Kondo state Sci. Rep. 623336

28 Rainis D, Trifunovic L, Klinovaja J and Loss D 2013 Towards a realistic transport modelling in a superconducting nanowire with Majorana fermions Phys. Rev. B 87024515

29 Chen H J and Zhu K D 2014 Nonlinear optomechanical detection for Majorana fermions via a hybrid nanomechanical system Nanoscale Res. Lett. 9166

30 Liu D E, Cheng M and Lutchyn R 2015 Probing Majorana physics in quantum-dot shot-noise experiments Phys. Rev. B $91081405(\mathrm{R})$

31 Hansen E B, Danon J and Flensberg K 2016 Phase-tunable Majorana bound states in a topological N-SNS junctions Phys. Rev. B 93094501

${ }^{32}$ Liu D E and Baranger H U 2011 Detecting a Majoranafermion zero mode using a quantum dot Phys. Rev. B $\mathbf{8 4}$ 201308(R)

33 Leijnse M and Flensberg K 2014 Thermoelectric signatures of a Majorana bound state coupled to a quantujm dot New J. Phys. 16015029

34 Seridonio A C, Siqueira E C, Desotti F A, Mchado R S and Yoshida M 2014 Fano interference and a slight fluctuation of the Majorana hallmark J. Appl. Phys. 115063706

35 Gong W -J, Zhang S -F, Li Z -C, Yi G and Zheng Y -S 2014 Detection of a Majorana fermion zero mode by a T-shaped quantum-dot structure Phys. Rev. B 89245413
36 Jiang C, Lu G and Gong W -J 2014 Tunable transport through a quantum dot chain with side-coupled Majorana bound states J. Appl. Phys. 116103704

37 Desotti F A, Ricco L S, de Souza M, Souza F M and Seridonio A C 2014 Probing the antisymmetric Fano interference assisted by a Majorana fermion J. Appl. Phys. 116173701

38 Stefański P 2015 Signatures of Majorana states in electron transport through a quantum dot coupled to topological wire Acta Phys. Polon. A 127198

39 Li Z -Z, Lam C -H and You J Q 2015 Probing Majorana bound states via counting statistics of a single electron transistor Sci. Rep. 511416

40 Chirla R and Moca C P 2016 Fingerprints of Majorana fermions in spin-resolved subgap spectroscopy Phys. Rev. B 94045405

41 Gong W -J, Zhang S -F, Li Z -C, Yi G and Zheng Y -S 2014 Andreev reflection in a T-shaped double-quantumdot structure induced by Majorana bound states J. Phys. Soc. Jpn. 83034706

42 Wang S -X, Li Y -X, Wang N and Liu J -J 2016 Andreev reflection in a T-shaped double quantum-dot with coupled Majorana bound states Acta Phys. Sin. 65137302

43 He J J, Ng T K and Law K T 2014 Selective equal-spin Andreev reflections induced by Majorana fermions Phys. Rev. Lett. 112037001

${ }^{44} \mathrm{Hu}$ L -H, Li C, Xu D -H, Zhou Y and Zhang F -C 2016 Theory for spin selective Andreev reflection in vortex core of topological superconductor: Majorana zero modes on spherical surface and application to spin polarized scanning tunneling microscope probe (arXiv:1607.03449)

45 Sun $\mathrm{H}-\mathrm{H}$ et al 2016 Majorana zero modes detected with spin selective Andreev reflection in the vortex of a topological superconductor Phys. Rev. Lett. 116257003

46 Mishmash R V, Aasen D, Higginbotham A P and Alicea J 2016 Approaching a topological phase transition in Majorana nanowires Phys. Rev. B 93245404

47 Barański J and Domański T 2011 Fano-type interference in quantum dots coupled between metallic and superconducting leads Phys. Rev. B 85205451 (2011).

48 Calle A M, Pacheco M and Orellana P A 2013 Fano effect and Andreev bound states in T-shape double quantum dots Phys. Lett. A 3771474

49 Nozaki D, Avdoshenko S M, Sevincli H and Cuniberti G 2014 Quantum interference in thermoelectric molecular junctions: A toy model perspective J. Appl. Phys. 116 074308

50 Trocha P and Barnaś J 2014 Spin-polarized Andreev transport influenced by Coulomb repulsion through a twoquantum-dot system Phys. Rev. B 89245418

51 Wójcik K P and Weymann I 2016 Thermopower of strongly correlated T-shaped double quantum dots Phys. Rev. B 93 085428

52 Sticlet D, Bena C and Simon P 2012 Spin and Majorana polarization in topological superconducting wires Phys. Rev. Lett. 108096802

53 Kjaergaard M, Wölms K and Flensberg K 2012 Majorana fermions in superconducting nanowires without spin-orbit coupling Phys. Rev. B 85020503

54 Shi Z C, Wang W and Yi X X 2016 Entangled states of two quantum dots mediated by Majorana fermions New J. Phys. 18023005

55 Vernek E, Penteado P H, Seridonio A C and Egues J C 2014 Suble leakage of a Majorana mode into a quantum dot Phys. Rev. B $8 \mathbf{9 1 6 5 3 1 4}$ 
56 Ruiz-Tijerina D A, Vernek E, Dias da Silva L G G V and Egues J C 2015 Interaction effects on a Majorana zero mode leaking into a quantum dot Phys. Rev. B 91115435

57 Balatsky A V, Vekhter I and Zhu J -X 2006 Impurityinduced states in conventional and unconventional superconductors Rev. Mod. Phys. 78373

58 Domański T 2010 Particle-hole mixing driven by the superconducting fluctuations Eur. Phys. J. B 74437

59 Golub A 2015 Multiple Andreev reflections in s-wave superconductor-quantum dot-topological superconductor tunnel junctions and Majorana bound states Phys. Rev. B 91205105

60 Žitko R 2011 Fano-Kondo effect in side-coupled double quantum dots at finite temperatures and the importance of two-stage Kondo screening Phys. Rev. B 81115316

61 Bauer J, Oguri A and Hewson A C 2007 Spectral properties of locally correlated electrons in a Bardeen Cooper Schrieffer superconductor J. Phys.: Condens. Matter 19 486211

62 Yamada Y, Tanaka Y and Kawakami N 2011 Interplay of Kondo and superconducting correlations in the nonequilibrium Andreev transport through a quantum dot Phys. Rev. B 84075484

63 Barański J and Domański T 2013 In-gap states of a quantum dot coupled between a normal and a superconducting lead J. Phys.: Condens. Matter 25435305

${ }^{64} \mathrm{Fu} \mathrm{L}$ and Kane C L 2009 Josephson current and noise at a superconductor/quantum-spin-Hall-insulator/superconductor junction Phys. Rev. B 79 161408(R)

65 Martín-Rodero A and Levy-Yeyati A 2011 Josephson and Andreev transport through quantum dots Adv. Phys. 60 899

66 Deacon R S, Tanaka Y, Oiwa A, Sakano R, Yoshida K, Shibata K, Hirakawa K and Tarucha S (2010) Kondoenhanced Andreev transport in single self-assembled InAs quantum dots contacted with normal and superconducting leads Phys. Rev. B 81 121308(R)

67 Hübler F, Wolf M J, Scherer T, Wang D, Beckmann D and Löhneysen H (2012) Observation of Andreev bound states at spin-active interfaces Phys. Rev. Lett. 109087004

${ }^{68}$ Chang W, Manucharyan V E, Jespersen T S, Nygård J and Marcus C M (2013) Tunneling spectroscopy of quasiparticle bound states in a spinful Josephson junction Phys. Rev. Lett. 110217005

${ }^{69}$ Lee E J H, Jiang X, Houzet M, Aguado R, Lieber Ch M and De Franceschi S (2014) Spin-resolved Andreev levels and parity crossings in hybrid superconductorsemiconductor nanostructures Nature Nanotechnology 979 